\title{
Exercise: an essential component of diabetes management
}

\author{
S Siyambalapitiya ${ }^{1}$, G Gunathilake ${ }^{1}$, I Perera ${ }^{2}$ \\ Sri Lanka Journal of Diabetes, Endocrinology and Metabolism 2012; 2: 57-60
}

\begin{abstract}
Objective: Regular exercise constitutes an essential component of diabetes management. However, the compliance with exercise related advice is poor among patients. Our objectives were to assess the compliance with exercise recommendations and the barriers to exercise among patients with diabetes.

Methods: 253 patients with type 2 diabetes treated in 3 different clinical settings; hospital diabetes clinic, hospital general medical clinics and general practitioners (GPs), were recruited for the study. Data were collected using an interviewer administered questionnaire.

Results: Out of 253 patients, only $45.1 \%(n=114)$ were properly educated regarding exercise as part of diabetes management. A higher percentage of patients in diabetes clinics were educated $(60.6 \%, n=63)$ compared to patients attending medical clinics $(26.5 \%, n=26)$ and the GPS $(49 \%, n=25)$ regarding exercise. However, the compliance with exercise recommendations is poor (11.5\%) irrespective of the clinical setting. Walking is the commonest mode of exercise $(72.4 \%, n=21)$ followed by exercise machines $(13.8 \%, n=4)$ and running $(13.8 \%, n=4)$. In the non-exercising group, majority $(51.6 \%, n=48)$ think that the activities of daily living are adequate as daily exercise. $18.3 \%(n=17)$ claim that they do not have time to exercise and $14 \%(n=13)$ are not really interested
\end{abstract}

Conclusions: Adherence to regular exercise as part of diabetes management is poor among patients with diabetes. Current health promotional and health information delivering techniques have also failed to achieve the desired attitude change among patients towards regular exercise. This highlights the need for novel approaches to address this problem.

\section{Introduction}

Type 2 diabetes is one of the commonest non communicable diseases and it is associated with significant morbidity and mortality. Current epidemiological data suggest that the prevalence of diabetes is on the rise and its negative economic impact is enormous $(1,2)$. With the genetic predisposition, unfavourable environmental contributors such as unhealthy dietary patterns and lack of exercise can lead to the development of diabetes in susceptible individuals. Physical inactivity that goes hand in hand with the economic development has been one of the most important reasons for this epidemiological transition. Reduction of insulin sensitivity is one of the main pathological processes of developing type 2 diabetes and lack of physical activity and obesity are the main contributors. Regular exercise improves insulin sensitivity, thereby the glycaemic control in patients with diabetes. Therefore, it is recommended as one of the most important treatment modalities in the management of diabetes.

Health benefits of exercise are enormous. In patients with diabetes, it improves the glycaemic control by increasing insulin sensitivity and glucose utilization and by decreasing the hepatic glucose production $(3,4)$. It can also prevent or delay the onset of diabetes in high risk individuals (5-7). It also helps in reducing weight in obese individuals. It reduces the cardiovascular co-morbidities such as hypertension, dyslipidaemia and improves the quality of life of diabetic patients. In order to get these benefits, it is recommended to carry out at least 150 minutes of exercise per week.

Although there is enough evidence to prove the benefits of exercise, initiating and maintaining regular physical activity remain a difficult challenge $(8,9)$. People in different communities have different individual, sociocultural and environmental reasons and barriers that hinder the adherence to regular exercise. Level of education and understanding regarding the importance of physical activity are important determinants for compliance with exercise $(10,11)$. Lack of interest $(10,12)$, lack of time $(10,13)$, depressive symptoms (14), physical limitations $(11,14)$, smoking (11), and female sex (14) are important patient factors. Fear of hypoglycaemia is one of the main reasons for not doing exercise among insulin-treated diabetics (15). Lack of social support from family and friends (14) is another important factor especially for elderly patients. Availability and accessibility to a proper place where physical activity can be carried out is another important

${ }^{1}$ North Colombo Teaching Hospital, Ragama, ${ }^{2}$ Community Dental Unit, Dental Institute, Maligawatta, Sri Lanka. 
environmental barrier $(12,13)$. Distraction from television is another noteworthy obstacle in some communities (13).

Although the barriers to carry out exercise are many, the factors that influence exercise practices in South Asian communities are largely unknown. The objectives of this study were to assess the knowledge regarding exercise, the degree of compliance to exercise recommendations and to find out reasons for non-adherence to exercise recommendations among Sri Lankan patients with diabetes.

\section{Method}

The study was conducted in North Colombo Teaching Hospital, Ragama (a tertiary care hospital). Ethical approval for the study was obtained from Faculty of Medicine, University of Kelaniya. Data collection was also done from 2 community clinics in 2 Medical Office of Health (MOH) areas, Ja-Ela and Katana. In order to obtain a proper cross-section of the diabetes population, we recruited a total number of 253 diabetic patients, 104 and 98 managed in the diabetes clinic and the medical clinics at North Colombo Teaching Hospital respectively, and 51 patients from the community (Katana and Ja-Ela $\mathrm{MOH}$ areas) who received treatment from the general practitioners (GPs). Patients from the diabetes clinic and medical clinics were selected randomly using the clinic registration numbers. Two clusters of patients receiving treatment from GPs were randomly selected from the 2 community clinics at Katana and Ja-Ela MOH area. After obtaining informed consent from the recruited patients, demographic data, data related to exercise level and the reasons for not exercising were collected using a pre-tested interviewer administered questionnaire.

\section{Results}

Distribution of the patient sample by type of care and socio-demographic characteristics is shown in Table 1. Out of 253 patients, $45.1 \%$ of participants were managed in the government tertiary care hospital diabetic clinic, another $41.1 \%$ in the government tertiary care hospital medical clinics and the rest $(13.8 \%)$ by the GPs in the community. Mean age of the participants was 57.03 years $( \pm 10.75)$ and the overwhelming majority $(80.3 \%)$ consisted of females. 193 (76.3\%) of the patients had above primary level (above grade 5) of education. However, only 17\% were employed.

Out of 253 patients, only $45.1 \%(n=114)$ had received proper education regarding exercise. Patients in the diabetic clinic were better informed $(60.6 \%, n=63)$ compared to patients attending medical clinics $(26.5 \%, n=26)$ and those managed by the GPs $(49 \%, n=25)$ regarding exercise. Irrespective of the clinical setting that they attended for treatment, the compliance with exercise advice has been poor (11.5\%) among patients with diabetes (Table 2). Walking is the commonest mode of exercise $(72.4 \%, \mathrm{n}=21)$ followed by work outs in exercise machines $(13.8 \%, n=4)$ and running $(13.8 \%, n=4)$. In the non-exercising group, majority of the patients $(51.6 \%, n=48)$ were under the impression that activities of daily living were adequate as daily exercise and they have not done any changes to improve their level of activity. $18.3 \%(n=17)$ claimed that they did not have the time to do exercise and 14\% (n=13) were not interested in doing any exercise. The rest (16.1\%, $\mathrm{n}=15$ ) did not carry out exercise due to physical disabilities such as back pain, joint pain, chest pain and age related disability (Table 2).

Table 1. Distribution of sample by place of care and socio-demographic attributes

\begin{tabular}{lc}
\hline & Total $\%$ \\
\hline Patient number & 253 \\
Hospital diabetic clinic & $114(45.1 \%)$ \\
Hospital medical clinics & $104(41.1 \%)$ \\
Private sector doctors & $35(13.8 \%)$ \\
Mean age (years) & $57.3( \pm 10.75)$ \\
Gender - Females & $203(80.2 \%)$ \\
Education level & \\
No education & $20(7.9 \%)$ \\
Up to grade 5 & $40(15.8 \%)$ \\
Grade 5-10 & $113(44.7 \%)$ \\
O/L and A/L & $79(31.2 \%)$ \\
University & $1(0.4 \%)$ \\
Employed & $43(17 \%)$
\end{tabular}

Table 2. Exercise related parameters in different health care settings and reasons for non-compliance to exercise

\begin{tabular}{lc}
\hline & No. $\%$ \\
\hline Patient received exercise advise & $114(45.1 \%)$ \\
Hospital diabetic clinic & $63(60.6 \%)$ \\
Hospital medical clinics & $26(26.5 \%)$ \\
Private sector doctors & $25(49.0 \%)$ \\
Patient actually doing exercise & $29(11.5 \%)$ \\
Hospital diabetic clinic & $15(14.4 \%)$ \\
Hospital medical clinics & $12(12.2 \%)$ \\
Private sector doctors & $2(3.9 \%)$ \\
Reasons not to exercise & \\
Lack of interest & $13(14.0 \%)$ \\
Lack of time & $17(18.3 \%)$ \\
Back pain & $5(5.4 \%)$ \\
Leg pain & $2(2.2 \%)$ \\
Arthritis & $3(3.2 \%)$ \\
Chest pain & $4(4.3 \%)$ \\
Age related disability & $1(1.1 \%)$ \\
Think that normal daily & \\
work is enough as exercise & $481.6 \%)$ \\
\hline
\end{tabular}




\section{Discussion}

We have evaluated a cross section of patients in the community, who attended different types of clinic settings for diabetes care. Significant proportion of patients had received information regarding the importance of regular exercise as part of diabetes management. However, a very small percentage of patients were actually carrying out regular exercise. As expected, the patients attending the diabetes clinics were better informed than those attending the general medical clinics and the GPs regarding the importance of regular exercise as a part of diabetes management. It was interesting to note that most of the patients in modern society with sedentary lifestyles believed that activities of daily living are more than adequate as exercise. These findings highlight the poor level of understanding regarding exercise and the importance of exercise as part of diabetes management among patients with diabetes. Current diabetes education strategies and techniques have failed to achieve the desired effects among patients in initiating and maintaining regular exercise. This highlights the need of novel approaches to achieve the desired attitude change among patients towards promoting exercise as part of diabetes management.

Despite knowing the importance of regular physical activity in the management of diabetes, most of the medical practitioners have failed to educate and re-enforce the patients to carry out regular physical activities. During busy patient consultations, priority is given to optimize and highlight the importance of drug treatment. However, due prominence is not given to this important management aspect and very limited time is devoted in educating and promoting exercise practices. This probably is one of the main reasons for our observations that need to be addressed properly in order to achieve the expected results.

Our study sample is dominated by elderly housewives (80.2\%). It could be speculated that they may be under the impression that engaging in day to day household chores provides adequate physical exercise. Moreover, there could be a plethora of socio-cultural and lifestyle factors especially among low and middle class females that influence their perceptions and attitudes regarding exercise in Sri Lankan context. Therefore, it is important that the health educators take these factors into consideration when delivering information regarding physical activity especially for overweight and obese housewives with diabetes. Walking has been the most accepted mode of exercise among our patients and this probably is the best mode of exercise that we could recommend. However, it is essential that these health care messages are tuned according to the level of health literacy of the individual patient and delivered in a way that are personally, socially and environmentally acceptable.
Although a reasonable proportion of patients received exercise advice as part of diabetes management, the desired outcome was not evident. This highlights the ineffectiveness of health education strategies and currently used health educating techniques such as health education leaflets and mass lectures that are carried out in our health care settings for diabetic patients. One to one advice tailored to individual needs has been the most effective especially for promoting exercise practices among patients $(16,17)$. However, it is time consuming, expensive and difficult to implement even in resource abundant settings. Several studies have shown promising results with the use of cheaper modes of communication such as mobile phones (18), internet (19) and social networking sites (20) in managing chronic diseases that can also be used for educating and promoting regular exercise in patients with diabetes. It is timely to make good use of these technological advances and find better novel cost effective solutions to suite different clinical settings.

Our study has shown that the compliance with exercise advice is poor among our patients despite receiving a reasonable degree of information regarding the importance of regular physical activity. This highlights the deficiencies and failures of current health educating strategies in healthcare settings to achieve the desired objectives. It is time to work out better cost effective ways of imparting this information aimed at attitudinal and behavioural changes in order to achieve better outcomes. Moreover, it is important to create health promoting settings with facilities to engage in exercise, which are easily accessible for the people to make "easy healthy choices".

\section{References}

1. Illangasekera U, Rambodagalla S, Tennakoon S. Temporal trends in the prevalence of diabetes mellitus in a rural community in Sri Lanka. The Journal of the Royal Society for the Promotion of Health 2004; 124: 92-4.

2. Katulanda P, Constantine GR, Mahesh JG, et al. Prevalence and projections of diabetes and pre-diabetes in adults in Sri Lanka - Sri Lanka Diabetes, Cardiovascular Study (SLDCS). Diabetic Medicine 2008; 25: 1062-9.

3. Hawley JA. Exercise as a therapeutic intervention for the prevention and treatment of insulin resistance. Diabetes Metabolism Research and Reviews 2004; 20: 383-93.

4. Fujita S, Rasmussen BB, Cadenas JG, et al. Aerobic exercise overcomes the age-related insulin resistance of muscle protein metabolism by improving endothelial function and Akt/ mammalian target of rapamycin signaling. Diabetes 2007; 56: $1615-22$.

5. Tuomilehto J, Lindstrom J, Eriksson JG, et al. Prevention of type 2 diabetes mellitus by changes in lifestyle among subjects with impaired glucose tolerance. New England Journal of Medicine 2001; 344: 1343-50. 
6. Pan XR, Li GW, Hu YH, et al. Effects of diet and exercise in preventing NIDDM in people with impaired glucose tolerance: the Da Qing IGT and Diabetes Study. Diabetes Care 1997; 20: 537-44.

7. Knowler WC, Barrett-Connor E, Fowler SE, et al. Reduction in the incidence of type 2 diabetes with lifestyle intervention or metformin. New England Journal of Medicine 2002; 346: 393-403.

8. Wing R, Goldstein M,Acton K, et al. Behavioral science research in diabetes: lifestyle changes related to obesity, eating behavior, and physical activity. Diabetes Care 2001; 24: 117-23.

9. Samaras K, Ashwell S, Mackintosh AM, et al. Will older sedentary people with non-insulin-dependent diabetes mellitus start exercising? A health promotion model. Diabetes Research and Clinical Practice 1997; 37: 121-8.

10. Donahue KE, Mielenz TJ, Sloane PD, et al. Identifying supports and barriers to physical activity in patients at risk for diabetes. Preventing Chronic Diseases 2006; 3: A119.

11. Wanko NS, Brazier CW, Young-Rogers D, et al. Exercise preferences and barriers in urban African Americans with type 2 diabetes. The Diabetes Educator 2004; 30: 502-13.

12. Shultz JA, Sprague MA, Branen LJ, Lambeth S. A comparison of views of individuals with type 2 diabetes mellitus and diabetes educators about barriers to diet and exercise. Journal of Health Communication 2001; 6: 99-115.

13. Thomas N, Alder E, Leese GP. Barriers to physical activity in patients with diabetes. Postgraduate Medical Journal 2004; 80: 287-91.
14. Satariano WA, Haight TJ, Tager IB. Reasons given by older people for limitation or avoidance of leisure time physical activity. Journal of the American Geriatric Society 2000; 48: 505-12.

15. Brazeau AS, Rabasa-Lhoret R, Strychar I, Mircescu H. Barriers to physical activity among patients with type 1 diabetes. Diabetes Care 2008; 31: 2108-9.

16. Bull FC, Kreuter MW, Scharff DP. Effects of tailored, personalized and general health messages on physical activity. Patient Education and Counselling 1999; 36: 181-92.

17. Kreuter MW, Bull FC, Clark EM, Oswald DL. Understanding how people process health information: a comparison of tailored and non-tailored weight-loss materials. Health Psychology 1999; 18: 487-94.

18. Lyles CR, Harris LT, Le T, Flowers J, Tufano J, Britt D, et al. Qualitative evaluation of a mobile phone and web-based collaborative care intervention for patients with type 2 diabetes. Diabetes Technology and Therapeutics 2011; 13: 563-9.

19. Ramadas A, Quek KF, Chan CK, Oldenburg B. Web-based interventions for the management of type 2 diabetes mellitus: a systematic review of recent evidence. International Journal of Medical Informatics 2011; 80: 389-405.

20. Greene JA, Choudhry NK, Kilabuk E, Shrank WH. Online social networking by patients with diabetes: a qualitative evaluation of communication with Facebook. Journal of General Internal Medicine 2011; 26: 287-92. 